\title{
INVESTIGACIONES
}

\section{Percepciones de la incursión de las TIC en la enseñanza superior en Ecuador}

\author{
The perception of the incursion of the TIC \\ in higher education in Ecuador
}

\author{
David A. Pacheco Montoya ${ }^{a}$, M. Esther Martínez-Figueira ${ }^{b}$ \\ ${ }^{a}$ Unidad Educativa Bilingüe Torremar de Ecuador. \\ dpacheco@torremar.edu.ec \\ ${ }^{b}$ Facultad de Ciencias de la Educación y del Deporte, Universidad de Vigo. \\ esthermf@uvigo.es
}

\section{RESUMEN}

Pese al aumento de las tecnologías de la información y comunicación (TIC) siguen vigentes preguntas como: ¿qué aportan en realidad al proceso de enseñanza-aprendizaje en la educación superior? y ¿cuál es el potencial de su uso? En ese contexto, se indagó la percepción del uso de las TIC que tienen estudiantes de Ciencias de la Educación de la Universidad Técnica Particular de Loja. Para ello, se realizó un estudio cuantitativo, descriptivo y transeccional, aplicando un cuestionario creado ad hoc y validado utilizando la técnica Delphi. Mediante el análisis estadístico de los datos se determinaron fortalezas y potencialidades en los conocimientos, usos y actitudes de los estudiantes frente a estas tecnologías, así como aspectos de mejora a tomarse en cuenta por profesores y autoridades. Ya que pese a la percepción positiva y uso habitual que tienen de las TIC, desconocen varias herramientas y aplicaciones tecnológicas específicas para fines educativos y laborales.

Palabras claves: educación mediática, tecnología educativa, formación del profesorado, pedagogía universitaria, enseñanza universitaria.

\begin{abstract}
Despite the growth of information and communication technologies (ICT), some questions remain, such as, how do information and communication technologies (ICT) actually contribute to the teaching-learning process in higher education, and what is the potential of their use? In this context, the perception of the use of ICT in students from the School of Education of the Universidad Técnica Particular de Loja was studied. For this purpose, a quantitative, descriptive, and trans-sectional study was carried out, applying an ad hoc questionnaire validated using the Delphi technique. Through the statistical analysis of data, the strengths and potentialities in the knowledge, uses, and attitudes of university students towards these technologies were defined, as well as the improvement issues that must be considered by professors and authorities. Despite the positive perception and regular use of ICT, many specific tools and technological applications for academic and work purposes are unknown to them.
\end{abstract}

Key words: media education, educative technology, teacher training, university pedagogy, university teaching. 


\section{INTRODUCCIÓN}

Actualmente, las universidades se encuentran ante grandes desafíos de parte de la sociedad, ya que incluso se ha llegado a cuestionar su necesidad en un mundo globalizado, cada vez más tecnológico, con mucha información disponible de forma libre, con la necesidad de personas que posean competencias personales y laborales muy flexibles, etc. Pese a ello, el número de estudiantes universitarios sigue en aumento, aunque no está claro si esto obedece a que las instituciones de educación superior han desarrollado nuevos modelos educativos o más bien de negocio, cuestionándose además si en verdad se está ofreciendo una educación de calidad o no (Cobo y Moravec, 2011). Como respuesta o consecuencia de estos desafíos, las tecnologías digitales están definiendo las nuevas formas de educación, lo cual amerita un análisis mucho más serio que el simple optimismo con que suele abordarse este tema, existiendo la necesidad de realizar más investigaciones que profundicen y problematicen la relación entre educación y tecnología (Cobo, 2016). En este sentido, algunos autores como González y Gutiérrez (2017) o Rodríguez (2010), resaltan directamente los problemas pedagógicos, afirmando que son escasos los resultados en la mejora de la enseñanza y el aprendizaje en las universidades que utilizan ampliamente las TIC, manteniéndose el desfase entre las habilidades desarrolladas y las requeridas en el mundo laboral. Por ello, sigue siendo válido plantearse la pregunta ¿qué aporta en realidad el uso de TIC a la educación superior?, cuya respuesta aún está pendiente.

Ahondando en esta problemática, según los informes regionales (Tarragona-España) del Informe Horizon del New Media Consortium (NMC) y la Universitat Oberta de Catalunya (Durall et al., 2012; Johnson et al., 2013), ya desde esos años las personas tenían la tendencia a trabajar, aprender y estudiar cuando quieran y desde donde quieran. Así como a usar medios sociales para interactuar, presentar ideas e información y juzgar la calidad de los contenidos y contribuciones, lo cual ha seguido incrementándose junto con el uso de los cursos en línea masivos y abiertos (MOOC), que ya se habrían convertido en una alternativa y suplemento a los cursos universitarios tradicionales. En los últimos dos años, el Informe Horizon continúa resaltando la tendencia al aprendizaje móvil y aunque con altibajos por los recursos que exigen, se perfilan con mucha fuerza el aprendizaje adaptativo y la analítica de aprendizaje, que aumentan la presión a las instituciones educativas y a los modelos de enseñanza-aprendizaje (Alexander et al., 2019; Brown et al., 2020).

En el Ecuador la tendencia es similar, aunque con sus particularidades y menor intensidad. Por ejemplo, en el informe Tecnologías de la Información y Comunicaciones del Instituto Nacional de Estadísticas y Censos (Instituto Nacional de Estadística y Censos, 2016) se reportó que el $78.9 \%$ de las personas entre 16 y 24 años de edad, y el 59.5\% de las personas entre 25 a 34 años de edad utilizan computadoras de escritorio y portátiles, pero solamente el $23.2 \%$ utiliza internet para actividades de educación y aprendizaje y apenas el $7.7 \%$ lo hace en una institución educativa. En términos generales, el principal recurso tecnológico cuya disponibilidad se ha incrementado es el internet móvil, con un aumento de 24 veces entre 2010 y 2017, lo cual se espera que tenga una gran incidencia en la educación durante los próximos años (Agencia de Regulación y Control de las Telecomunicaciones, 2017).

Centrando el análisis en los estudiantes universitarios, existe poca información respecto a qué TIC utilizan, cómo lo hacen, con qué propósito y si requieren formación específica en ellas o no, lo cual es indispensable para gestionar el accionar docente, por ejemplo sus estrategias de enseñanza y procesos de evaluación. Al respecto, García (2015) realizó 
un estudio para tratar de conocer la realidad de los estudiantes que se integran a la vida universitaria en la modalidad de estudios a distancia de la Universidad Técnica Particular de Loja (UTPL), en donde se resaltaba la importancia de su adaptación para la culminación exitosa de la carrera, evidenciándose la falta de competencias digitales relacionadas con aspectos como la necesidad de obtener información administrativa y académica, y de comunicarse entre compañeros y con los docentes. Así mismo, en un estudio que vinculó la educación superior virtual con el aprendizaje colaborativo y comparó tres ambientes educativos, dos de Ecuador y uno de España, Herrera (2019) encontró que existe resistencia en los estudiantes y docentes ecuatorianos para participar en procesos de construcción colaborativa de conocimiento usando tecnologías. Afortunadamente, en el mismo estudio se evidenció que luego de la implementación de estas experiencias en las denominadas aulas virtuales, las acogieron positivamente.

Es por ello, que el desarrollo de las denominadas competencias digitales se inicia con la percepción y actitud que tienen los estudiantes y profesores frente a las mismas, ya que, si se considera que alguna herramienta o aplicación tecnológica no es útil, no tendrán ningún interés en aprender a usarla. Lo mismo ocurriría con ciertas tecnologías que, aunque el estudiante piense que son interesantes, no se profundizará en su estudio si no son requeridas por los docentes y en los programas académicos. Convirtiéndose en fundamental el comportamiento y las decisiones de los profesores y autoridades frente a las TIC, lo cual dependerá en gran medida de su nivel de conocimientos pedagógicos y tecnológicos (Ortiz, Peñaherrera y Ortega, 2012).

A pesar de que los estudiantes consideran que las TIC están cada vez más presentes en el día a día de las actividades académicas, en una investigación con un análisis cualitativo realizada por González y Gutiérrez (2017), se determinó que ellos tienen la percepción de que la frecuencia de uso no es sistemática, disminuyendo por ello el nivel de aprendizaje posible. Además, mientras los estudiantes están motivados para trabajar con la denominada web 2.0, muchos profesores aún utilizan la web 1.0, impidiendo con ello un uso adecuado de las TIC en la educación superior. Esta situación demuestra la urgencia de la capacitación docente de forma que sepan cómo crear situaciones de aprendizaje en donde se dé ejemplo del buen uso de las TIC, especialmente si se desempeñan como tutores.

En un estudio realizado por Torres-Coronas y Vidal-Blasco (2015) se evidenció la influencia de este desajuste entre los usos de las TIC que se aprenden en la universidad y los que se requieren en el futuro laboral, encontrándose diferencias entre las percepciones de los estudiantes con las de los empleadores, quienes en casi todos los aspectos analizados tuvieron una percepción de las competencias digitales por debajo de la de los estudiantes. En este mismo estudio se pudo determinar que las mujeres tenían una mejor percepción de su competencia digital en el uso de redes sociales y sobre aspectos legales y de seguridad en la red, mientras que los hombres se destacaron en la comunicación en línea. Además, se encontró que los estudiantes de ingenierías fueron superiores en aspectos como el hardware y la comunicación en línea, a diferencia de los estudiantes de ciencias sociales que lo hicieron en redes sociales y en los aspectos legales y de seguridad.

La adecuada implementación de sistemas de evaluación por competencias es otro de los aspectos que deben considerarse para la integración y el desarrollo de las TIC en la docencia universitaria, lo cual también depende en primera instancia de las percepciones y actitudes de los docentes. Ya que como lo afirman Hamodi, López y López (2015), el tipo de evaluación condiciona el qué y el cómo aprenden los estudiantes. Incluso se considera 
que la evaluación es "el arma más poderosa que tienen los profesores para influir en el modo en el que los estudiantes responden a las asignaturas y se comportan como alumnos" (Hamodi et al., 2015, p. 61). Sin embargo, la evaluación formativa que es la que se debería aplicar durante el desarrollo de competencias, en este caso digitales, aún no ha pasado sustancialmente de la teoría a la práctica pedagógica, en parte porque los mismos docentes no confían en que su formación profesional los haga competentes en el uso didáctico de las TIC (Palacios y López-Pástor, 2013; Cabero-Almenara y Martínez, 2019).

Recurriendo a un análisis más amplio, se considera que la integración de las TIC en las instituciones de educación superior tanto a nivel docente como estudiantil, pese a haberse iniciado hace varias décadas con una diversidad de enfoques, usos y aplicaciones, deja como evidencia que las habilidades y prácticas de algunos estudiantes aún están restringidas al uso del correo electrónico y la búsqueda arbitraria de información en internet, debido a que en muchos casos se han creado aulas de informática pero no se ha llevado la informática al aula (Cabero, 2010; Perazzo, 2008). Y cada vez el problema se agrava más, ya que las nuevas concepciones como el aprendizaje a lo largo de la vida, aprender a aprender, aprendizaje móvil, aprendizaje adaptativo, etc., obligan a que las universidades revisen si realmente están atendiendo las necesidades de aprendizaje de los estudiantes (Rodríguez, 2010; Cabero-Almenara y Martínez, 2019; Alexander et al., 2019; Brown et al., 2020).

Es lamentable que esto haya ocurrido, a pesar que desde el surgimiento de las TIC los especialistas tenían claro tanto la complejidad como el potencial de desarrollar y alcanzar la competencia digital, especialmente en ambientes formales de aprendizaje en donde debían incorporarse de forma planificada, sistemática e integrada a los procesos educativos en marcha. Es así que Gros y Contreras (2006) plantearon tempranamente junto a la definición de alfabetización digital, una amplia lista de habilidades o características que ahora corresponderían a la competencia digital. Más tarde la Organización de las Naciones Unidas para la Educación, la Ciencia y la Cultura (2011) publicó los estándares en competencias TIC para docentes, en donde se incluyen la mayoría de esas mismas características y se han convertido en una de las principales referencias internacionales. Allí se resalta: buscar, analizar y evaluar información; solucionar problemas y tomar decisiones; ser usuarios creativos y eficaces de herramientas de productividad; ser comunicadores, colaboradores, publicadores y productores de información; y ciudadanos informados para contribuir responsablemente a la sociedad.

En estas circunstancias, la actual sociedad del conocimiento obliga a plantearse nuevas ideas y concepciones sobre el aprendizaje y las estrategias para alcanzarlo, de manera especial en el desarrollo de competencias digitales en los docentes en formación, quienes luego influirán en las nuevas generaciones de estudiantes. Al respecto, Cabero-Almenara y Martínez (2019) plantean un análisis y proponen la necesidad de un proceso de formación del profesorado en TIC mediante las etapas de iniciación, incorporación y transformación con 5 dimensiones en cada una; ya que como señalan Martínez y Raposo (2006): "si los estudiantes universitarios, futuros profesionales en formación, utilizan y manejan las TIC también lo harán durante el desempeño de su profesión" (p. 175). Parte de la solución sería modificar los proyectos curriculares de forma que los estudiantes puedan alcanzar no solamente competencias digitales sino también mediáticas, desarrollando un desempeño crítico en la sociedad digital y más exitoso en el mundo laboral (González y Gutiérrez, 2017). Por ello, los currículos deberán ser de calidad, coherentes con los requerimientos actuales y revisados anualmente (Torres-Coronas y Vidal-Blasco, 2015). 


\section{MÉTODO}

En este estudio que tuvo un enfoque cuantitativo, se utilizó la técnica de la encuesta y se recolectó la información mediante el cuestionario denominado "Percepción del estudiante universitario acerca del uso, integración y competencias en TIC (CUIC-TIC-EU)", creado para esta investigación usando como referencia los de Prendes (2010) y Raposo et al. (2012); que fue validado por cinco expertas en educación superior, utilizando la técnica denominada "Delphi modificada" incluyendo una prueba piloto, que ha demostrado ser apropiada para estudios en el ámbito educativo (Cabero, 2014; George y Trujillo, 2018; López-Gómez, 2018). Los datos obtenidos fueron analizados mediante técnicas estadísticas usando el programa SPSS.

El diseño de investigación fue de tipo no experimental, con un carácter transversal, recogiéndose la información durante el ciclo académico octubre 2016 - febrero 2017. Así mismo, tuvo un carácter descriptivo buscando responder las preguntas de investigación que abarcaban temas como: tipo de uso de las TIC, forma de interacción con ellas, recursos personales movilizados para su uso, percepción sobre las competencias actuales y requeridas, y las necesidades formativas respecto a las TIC.

La población investigada fueron los 40 estudiantes de la carrera de Ciencias de la Educación de la Universidad Técnica Particular de Loja, modalidad abierta y a distancia, matriculados en el periodo académico octubre 2016 - febrero 2017, pertenecientes al Centro Universitario de la ciudad de Guayaquil, a partir de la cual mediante un muestreo no probabilístico, se obtuvo una muestra conformada por 33 estudiantes, quienes a su vez conformaron la unidad de análisis al responder el cuestionario, permitiendo la posterior descripción y análisis del uso que hacen de las TIC en sus estudios. La aplicación del cuestionario se realizó durante las evaluaciones presenciales parciales de ese periodo académico, autoadministrado en forma individual. La variable analizada fue la percepción que tienen los estudiantes universitarios acerca de las TIC, considerando las dimensiones: frecuencia de uso, nivel de conocimiento, importancia, actitud y necesidad de formación.

\section{RESULTADOS}

De los 33 estudiantes que resolvieron el cuestionario, 20 fueron mujeres y 13 hombres, y el rango de edad fue de 18 a 51 años. Los resultados se organizaron de acuerdo a las preguntas de investigación, resaltando las principales respuestas tanto positivas como negativas, organizadas en una escala de 1 a 4 , en donde 1 significa nada/totalmente en desacuerdo y 4 significa mucho/totalmente de acuerdo. En esta ocasión se presentan los resultados respecto al nivel de conocimientos que poseen los estudiantes sobre las diferentes herramientas, recursos y aplicaciones tecnológicas, y su frecuencia de uso para el aprendizaje.

Como se puede observar en la Figura 1, las principales herramientas, recursos y aplicaciones tecnológicas conocidas "bastante" y "mucho" por los estudiantes universitarios son: los navegadores de internet con el $93.94 \%$, el correo electrónico con el $87.88 \%$ y las redes sociales con el $84.85 \%$. Por el contrario, las referidas como "poco" y "nada" conocidas son: la creación de páginas web con el $87.88 \%$, las bases de datos bibliográficas con el $84.85 \%$ y la edición on-line de texto con el $81.82 \%$. De forma que los estudiantes universitarios que participaron en este estudio tendrían un conocimiento de las TIC similar 
a las demás personas, ya que no está específicamente orientado hacia las necesidades académicas, sino más bien para la búsqueda de información diversa y la interacción con otras personas. Esta idea se refuerza con el poco conocimiento que indicaron tener sobre plataformas educativas, bases de datos en general, y particularmente de las bibliográficas, aspectos que deberían ser fundamentales para sus estudios universitarios, ya que representan fuentes de información más confiables. Así mismo, se aprecia que la mayoría aún no han tenido la necesidad de conocer cómo producir textos o generar contenidos propios para compartirlos en los medios tecnológicos, y son pocos los que conocen el uso de las TIC para aplicar estrategias de estudio como los mapas conceptuales que potencian el aprendizaje, lo cual debería ser promovido por los docentes.

Figura 1. Conocimiento que tienen los estudiantes universitarios sobre herramientas, recursos y aplicaciones tecnológicas para su aprendizaje.

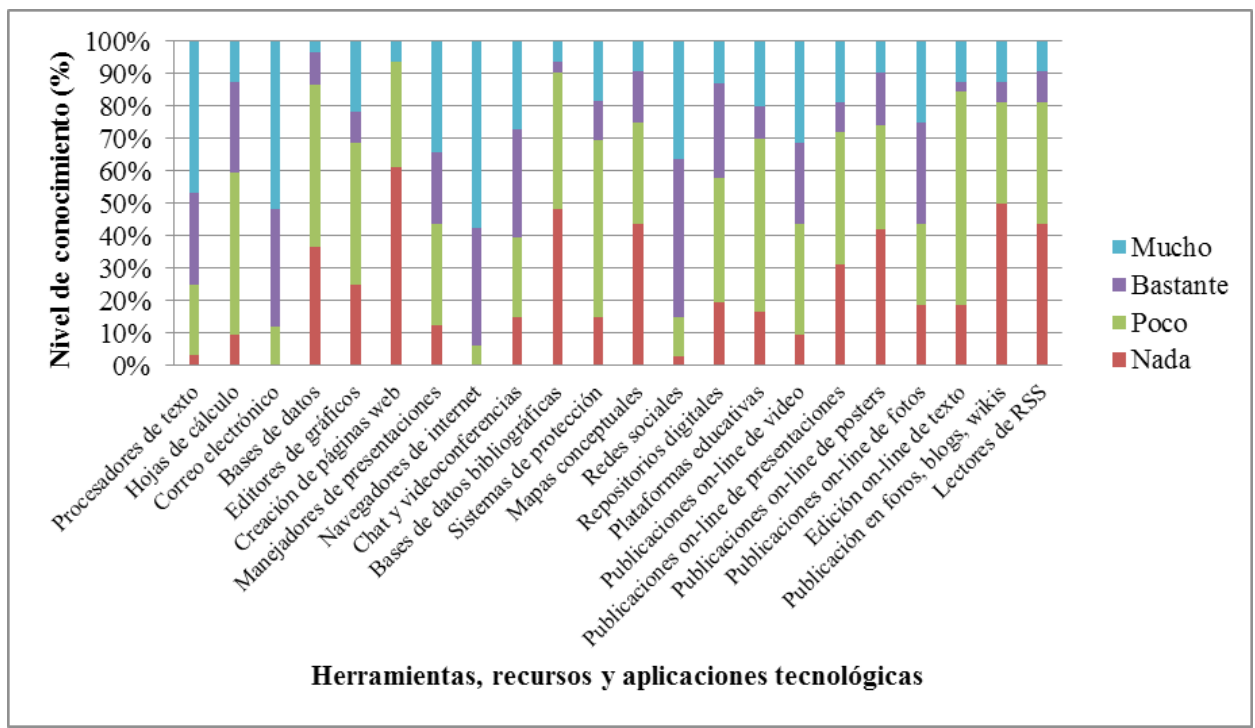

Fuente: elaboración propia.

Para el análisis por edad se establecieron cuatro grupos: de 18 a 22 años, de 23 a 28 años, de 29 a 35 años y de 36 a 51 años, y se calcularon los promedios de los valores asignados a las respuestas, en una escala de 1 a 4. Luego, se aplicó un análisis de correlación no paramétrica utilizando el estadístico rho de Spearman, para establecer si existían algunas correlaciones significativas al nivel de 0.05 y unilaterales. De esta forma, se pudo determinar que quienes tienen entre 23 y 28 años creen poseer un mayor conocimiento sobre herramientas, recursos y aplicaciones tecnológicas para su aprendizaje, con un valor promedio de 2.66. Al contrario, el grupo de estudiantes comprendido entre 36 y 51 años de edad, creen poseer un menor conocimiento en estos mismos aspectos, con un valor promedio de 1.93. Con estos resultados se encontraron correlaciones negativas significativas entre el aumento de la edad y el nivel de conocimientos sobre procesadores de 
texto, manejadores de presentaciones, navegadores de internet, redes sociales, repositorios digitales y publicaciones on-line de fotos.

Tabla 1. Relación entre la edad y el nivel de conocimientos que tienen los estudiantes universitarios sobre herramientas, recursos y aplicaciones tecnológicas para su aprendizaje

\begin{tabular}{|l|c|c|c|c|c|}
\hline $\begin{array}{l}\text { Herramientas, recursos } \\
\text { y aplicaciones }\end{array}$ & $\mathrm{N}$ & $\begin{array}{c}\text { Media } \\
\text { (nivel de } \\
\text { conocimiento) }\end{array}$ & $\begin{array}{c}\text { Desviación } \\
\text { estándar }\end{array}$ & $\begin{array}{c}\text { Coeficiente de } \\
\text { correlación } \\
\text { (Rho de Spearman) }\end{array}$ & $\begin{array}{c}\text { Significatividad } \\
\text { (unilateral) }\end{array}$ \\
\hline Procesador de texto & 32 & 3.16 & 0.99 & -0.465 & 0.004 \\
\hline Hoja de cálculo & 32 & 2.41 & 0.91 & -0.224 & 0.108 \\
\hline Correo electrónico & 32 & 3.41 & 0.71 & -0.252 & 0.082 \\
\hline Base de datos & 32 & 1.66 & 0.90 & -0.003 & 0.494 \\
\hline Editor de gráficos & 32 & 2.25 & 1.14 & -0.143 & 0.217 \\
\hline $\begin{array}{l}\text { Creación de páginas } \\
\text { web }\end{array}$ & 32 & 1.44 & 0.88 & -0.112 & 0.271 \\
\hline $\begin{array}{l}\text { Manejador de } \\
\text { presentaciones }\end{array}$ & 32 & 2.75 & 1.14 & -0.322 & 0.036 \\
\hline Navegador de internet & 32 & 3.53 & 0.62 & -0.338 & 0.029 \\
\hline Chat y videoconferencia & 32 & 2.72 & 1.05 & -0.170 & 0.176 \\
\hline $\begin{array}{l}\text { Bases de datos } \\
\text { bibliográficas }\end{array}$ & 32 & 1.53 & 0.88 & 0.071 & 0.350 \\
\hline Sistemas de protección & 32 & 2.31 & 0.97 & -0.203 & 0.133 \\
\hline Mapas conceptuales & 32 & 1.81 & 1.03 & 0.053 & 0.387 \\
\hline Redes sociales & 32 & 3.19 & 0.78 & -0.517 & 0.001 \\
\hline Repositorios digitales & 32 & 2.25 & 1.08 & -0.528 & 0.001 \\
\hline Plataformas educativas & 32 & 2.19 & 1.12 & -0.250 & 0.084 \\
\hline $\begin{array}{l}\text { Publicaciones on-line } \\
\text { de video }\end{array}$ & 32 & 2.69 & 1.12 & -0.153 & 0.202 \\
\hline $\begin{array}{l}\text { Publicaciones on-line } \\
\text { de presentaciones }\end{array}$ & 32 & 2.13 & 1.13 & -0.147 & 0.211 \\
\hline $\begin{array}{l}\text { Publicaciones on-line } \\
\text { de posters }\end{array}$ & 32 & 1.84 & 1.08 & -0.189 & 0.150 \\
\hline $\begin{array}{l}\text { Publicaciones on-line } \\
\text { de fotos }\end{array}$ & 32 & 2.59 & 1.13 & -0.382 & 0.015 \\
\hline Edición on-line de texto & 32 & 2.06 & 0.91 & -0.240 & 0.093 \\
\hline $\begin{array}{l}\text { Publicación en foros, } \\
\text { blogs, wikis }\end{array}$ & 32 & 1.78 & 1.07 & -0.054 & -0.16 \\
\hline Lectores de RSS & 32 & 1.81 & 1.00 & & 0.384 \\
\hline
\end{tabular}

Nota: Las correlaciones significativas al nivel de 0.05 (unilateral) están sombreadas.

Fuente: elaboración propia. 
En el análisis por sexo, utilizando la misma escala de respuestas, se aplicó la prueba no paramétrica de chi cuadrada, para determinar si habían o no diferencias significativas al nivel de 0.05 , encontrándose que los hombres consideran que su nivel de conocimientos es mayor que el de las mujeres en: procesadores de texto, hojas de cálculo, correo electrónico, bases de datos, navegadores de internet, programas de chat y videoconferencias, sistemas de protección, repositorios digitales, publicaciones on-line de video, publicaciones on-line de presentaciones, publicaciones on-line de posters, edición on-line de texto y lectores de RSS.

Tabla 2. Prueba de chi cuadrada para la relación entre el sexo y el conocimiento que tienen los estudiantes universitarios sobre herramientas, recursos y aplicaciones tecnológicas para su aprendizaje

\begin{tabular}{|l|c|c|c|c|c|}
\hline \multirow{2}{*}{$\begin{array}{l}\text { Herramientas, recursos y } \\
\text { aplicaciones }\end{array}$} & \multirow{2}{*}{$\begin{array}{c}\text { Valor promedio } \\
\text { hombres }\end{array}$} & $\begin{array}{c}\text { Valor promedio } \\
\text { mujeres }\end{array}$ & \multicolumn{2}{|c|}{ Chi cuadrada de Pearson } \\
\cline { 5 - 7 } & 3.75 & 2.85 & 9.545 & 4 & 0.049 \\
\hline Procesador de texto & 3.08 & 2.05 & 16.013 & 4 & 0.003 \\
\hline Hoja de cálculo & 3.77 & 3.15 & 6.329 & 2 & 0.042 \\
\hline Correo electrónico & 2.17 & 1.56 & 10.763 & 4 & 0.029 \\
\hline Base de datos & 2.92 & 1.90 & 8.592 & 4 & 0.072 \\
\hline Editor de gráficos & 1.58 & 1.47 & 0.242 & 3 & 0.971 \\
\hline Creación de páginas web & 3.42 & 2.40 & 8.457 & 4 & 0.076 \\
\hline Manejador de presentaciones & 3.85 & 3.30 & 6.620 & 2 & 0.037 \\
\hline Navegador de internet & 3.46 & 2.25 & 11.397 & 3 & 0.010 \\
\hline Chat y videoconferencia & 2.00 & 1.47 & 5.088 & 4 & 0.278 \\
\hline Bases de datos bibliográficas & 2.92 & 1.95 & 8.567 & 3 & 0.036 \\
\hline Sistemas de protección & 2.00 & 1.85 & 3.342 & 4 & 0.502 \\
\hline Mapas conceptuales & 3.54 & 2.95 & 6.124 & 3 & 0.106 \\
\hline Redes sociales & 2.92 & 2.00 & 10.313 & 4 & 0.035 \\
\hline Repositorios digitales & 2.75 & 2.06 & 5.339 & 4 & 0.254 \\
\hline Plataformas educativas & 3.58 & 2.30 & 14.637 & 4 & 0.006 \\
\hline Publicaciones on-line de video & 2.83 & 1.75 & 10.060 & 4 & 0.039 \\
\hline Publicaciones on-line de & 2.58 & 1.53 & 11.961 & 4 & 0.018 \\
\hline presentaciones & 2.33 & 1.50 & 7.084 & 4 & 0.132 \\
\hline Publicaciones on-line de posters & 2.25 & 8.393 & 4 & 0.078 \\
\hline Publicaciones on-line de fotos & 1.70 & 13.454 & 4 & 0.009 \\
\hline Edición on-line de texto & & 1.35 & 16.894 & 4 & 0.002 \\
\hline Publicación en foros, blogs, wikis & & & & \\
\hline Lectores de RSS & & & & & \\
\hline
\end{tabular}

Nota: Las correlaciones significativas al nivel de 0.05 (bilateral) están sombreadas.

Fuente: elaboración propia. 
Respecto a la frecuencia de uso de las TIC que hacen los estudiantes en su aprendizaje se encontró que las herramientas, recursos y aplicaciones tecnológicas usadas "bastante" y "mucho" por los estudiantes son: los navegadores de internet con el $87.88 \%$, el correo electrónico con el $78.79 \%$ y las redes sociales con el 78.79\%. Y las usadas "poco" y "nada" son: los lectores de RSS con el $84.85 \%$, los mapas conceptuales con el $84.85 \%$ y la creación de páginas web con el $81.82 \%$. Estos resultados concuerdan parcialmente con los obtenidos respecto al conocimiento de las TIC, ya que no se destaca su uso en actividades académicas, ni se evidencia un uso frecuente de herramientas útiles para obtener información específica y mejorar las estrategias de estudio. En la Figura 2 se pueden observar los resultados completos de este aspecto.

Figura 2. Frecuencia de uso que hacen los estudiantes universitarios de las herramientas, recursos y aplicaciones tecnológicas para su aprendizaje.

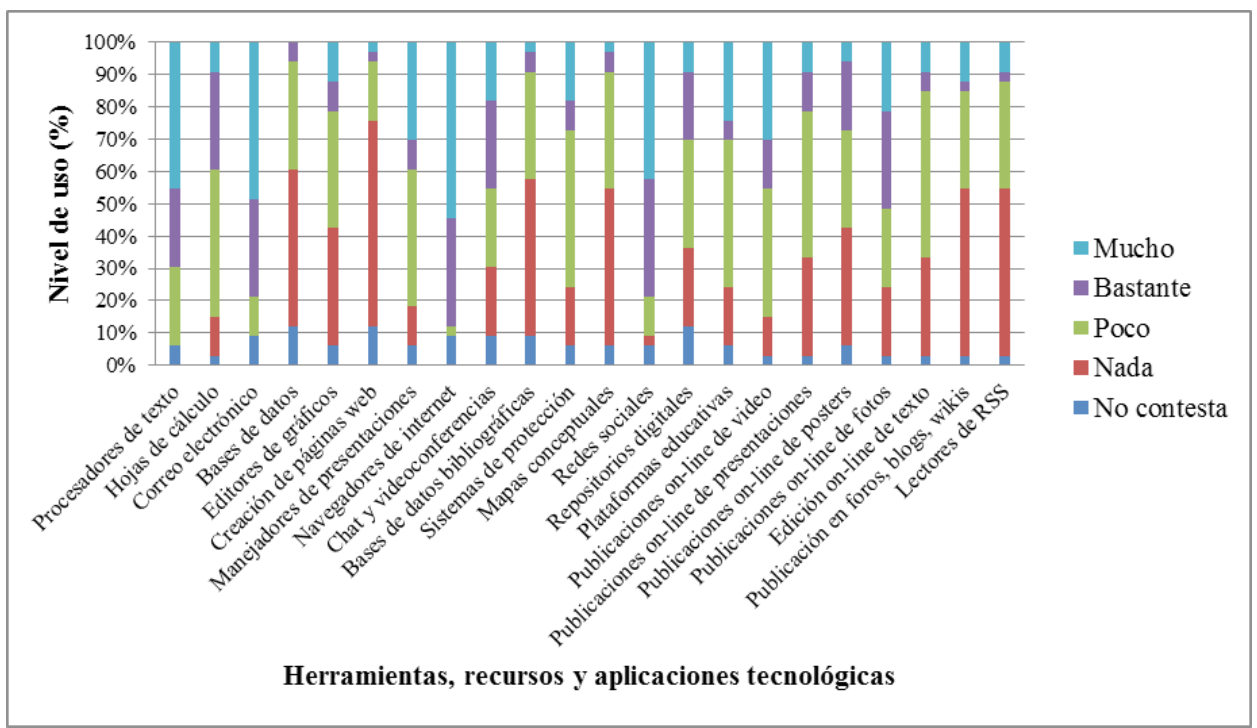

Fuente: elaboración propia.

En el análisis por edad se encontró que quienes están en un rango entre 23 y 28 años tienen un mayor nivel de uso, con un promedio de 2.57, a diferencia del grupo que comprende las edades entre 36 y 52 años, quienes presentaron un promedio de 1.90, indicando que hacen menor uso de estas TIC. Así mismo, se determinaron correlaciones negativas significativas entre el aumento de la edad y el uso de correo electrónico, manejadores de presentaciones, navegadores de internet, sistemas de protección, redes sociales, repositorios digitales y publicaciones on-line de fotos. 
Estudios Pedagógicos XLVII N²: 99-116, 2021

PERCEPCIONES DE LA INCURSIÓN DE LAS TIC EN LA ENSEÑANZA SUPERIOR EN ECUADOR

Tabla 3. Relación entre la edad y el uso que hacen los estudiantes universitarios de las herramientas, recursos y aplicaciones tecnológicas para su aprendizaje

\begin{tabular}{|c|c|c|c|c|c|}
\hline $\begin{array}{l}\text { Herramientas, } \\
\text { recursos y aplicaciones }\end{array}$ & $\mathrm{N}$ & $\begin{array}{c}\text { Media } \\
\text { (nivel de uso) }\end{array}$ & $\begin{array}{l}\text { Desviación } \\
\text { estándar }\end{array}$ & $\begin{array}{c}\text { Coeficiente de } \\
\text { correlación } \\
\text { (Rho de Spearman) }\end{array}$ & $\begin{array}{l}\text { Significatividad } \\
\quad \text { (unilateral) }\end{array}$ \\
\hline Procesador de texto & 32 & 3.13 & 1.01 & -0.156 & 0.197 \\
\hline Hoja de cálculo & 32 & 2.38 & 0.83 & 0.124 & 0.249 \\
\hline Correo electrónico & 32 & 3.19 & 1.09 & -0.481 & 0.003 \\
\hline Base de datos & 32 & 1.38 & 0.75 & 0.120 & 0.257 \\
\hline Editor de gráficos & 32 & 1.91 & 1.06 & -0.063 & 0.367 \\
\hline Creación de páginas web & 32 & 1.25 & 0.80 & -0.010 & 0.478 \\
\hline Manejador de presentaciones & 32 & 2.53 & 1.16 & -0.332 & 0.032 \\
\hline Navegador de internet & 32 & 3.34 & 1.04 & -0.457 & 0.004 \\
\hline Chat y videoconferencia & 32 & 2.31 & 1.20 & -0.209 & 0.125 \\
\hline Bases de datos bibliográficas & 32 & 1.50 & 0.84 & -0.098 & 0.296 \\
\hline Sistemas de protección & 32 & 2.22 & 1.07 & -0.316 & 0.039 \\
\hline Mapas conceptuales & 32 & 1.56 & 0.80 & -0.114 & 0.267 \\
\hline Redes sociales & 32 & 3.16 & 0.99 & -0.500 & 0.002 \\
\hline Repositorios digitales & 32 & 1.97 & 1.12 & -0.369 & 0.019 \\
\hline Plataformas educativas & 32 & 2.31 & 1.15 & -0.069 & 0.353 \\
\hline $\begin{array}{l}\text { Publicaciones on-line de } \\
\text { video }\end{array}$ & 32 & 2.66 & 1.07 & -0.183 & 0.158 \\
\hline $\begin{array}{l}\text { Publicaciones on-line de } \\
\text { presentaciones }\end{array}$ & 32 & 2.00 & 0.92 & -0.152 & 0.203 \\
\hline $\begin{array}{l}\text { Publicaciones on-line de } \\
\text { posters }\end{array}$ & 32 & 1.91 & 1.00 & -0.180 & 0.162 \\
\hline $\begin{array}{l}\text { Publicaciones on-line de } \\
\text { fotos }\end{array}$ & 32 & 2.53 & 1.08 & -0.310 & 0.042 \\
\hline Edición on-line de texto & 32 & 1.94 & 0.88 & -0.288 & 0.055 \\
\hline $\begin{array}{l}\text { Publicación en foros, blogs, } \\
\text { wikis }\end{array}$ & 32 & 1.75 & 1.02 & -0.032 & 0.430 \\
\hline Lectores de RSS & 32 & 1.69 & 0.93 & -0.006 & 0.487 \\
\hline
\end{tabular}

Nota: Las correlaciones significativas al nivel de 0.05 (unilateral) están sombreadas.

Fuente: elaboración propia. 
En cuanto a la percepción del nivel de uso de las TIC para su aprendizaje, el análisis estadístico por sexo mostró diferencias significativas solamente en los programas de chat y videoconferencias, y en las publicaciones on-line de video; indicando que los hombres los usan más que las mujeres.

Tabla 4. Prueba de chi cuadrada para la relación entre el sexo y el uso que hacen los estudiantes universitarios de las herramientas, recursos y aplicaciones tecnológicas para su aprendizaje

\begin{tabular}{|c|c|c|c|c|c|}
\hline \multirow[b]{2}{*}{$\begin{array}{l}\text { Herramientas, } \\
\text { recursos y aplicaciones }\end{array}$} & \multirow[b]{2}{*}{$\begin{array}{c}\text { Valor } \\
\text { promedio } \\
\text { hombres }\end{array}$} & \multirow[b]{2}{*}{$\begin{array}{c}\text { Valor } \\
\text { promedio } \\
\text { mujeres }\end{array}$} & \multicolumn{3}{|c|}{ Chi cuadrada de Pearson } \\
\hline & & & Valor & $\mathrm{gl}$ & $\begin{array}{l}\text { Sig. asintótica } \\
\text { (bilateral) }\end{array}$ \\
\hline Procesador de texto & 3.46 & 3.06 & 5.845 & 3 & 0.119 \\
\hline Hoja de cálculo & 2.77 & 2.11 & 7.869 & 4 & 0.096 \\
\hline Correo electrónico & 3.67 & 3.22 & 3.402 & 3 & 0.334 \\
\hline Base de datos & 1.62 & 1.44 & 5.846 & 3 & 0.119 \\
\hline Editor de gráficos & 2.31 & 1.72 & 4.728 & 4 & 0.316 \\
\hline Creación de páginas web & 1.54 & 1.25 & 5.875 & 4 & 0.209 \\
\hline Manejador de presentaciones & 3.00 & 2.33 & 5.047 & 4 & 0.283 \\
\hline Navegador de internet & 3.75 & 3.44 & 2.221 & 3 & 0.528 \\
\hline Chat y videoconferencia & 3.08 & 1.94 & 11.127 & 4 & 0.025 \\
\hline Bases de datos bibliográficas & 1.83 & 1.67 & 2.293 & 4 & 0.682 \\
\hline Sistemas de protección & 2.83 & 1.95 & 6.822 & 4 & 0.146 \\
\hline Mapas conceptuales & 1.77 & 1.50 & 2.983 & 4 & 0.561 \\
\hline Redes sociales & 3.58 & 3.05 & 4.030 & 4 & 0.402 \\
\hline Repositorios digitales & 2.54 & 1.88 & 8.114 & 4 & 0.087 \\
\hline Plataformas educativas & 2.54 & 2.28 & 1.866 & 4 & 0.760 \\
\hline Publicaciones on-line de video & 3.38 & 2.16 & 11.607 & 4 & 0.021 \\
\hline Publicaciones on-line de presentaciones & 2.54 & 1.63 & 9.196 & 4 & 0.056 \\
\hline Publicaciones on-line de posters & 2.38 & 1.67 & 8.787 & 4 & 0.067 \\
\hline Publicaciones on-line de fotos & 2.77 & 2.37 & 3.501 & 4 & 0.478 \\
\hline Edición on-line de texto & 2.38 & 1.63 & 9.421 & 4 & 0.051 \\
\hline Publicación en foros, blogs, wikis & 2.08 & 1.53 & 4.605 & 4 & 0.330 \\
\hline Lectores de RSS & 2.23 & 1.32 & 8.765 & 4 & 0.067 \\
\hline
\end{tabular}

Nota: Las correlaciones significativas al nivel de 0.05 (bilateral) están sombreadas.

Fuente: elaboración propia. 
Adicionalmente, como se muestra en la Figura 3, se encontró que las actividades académicas en las que los estudiantes universitarios más utilizan las TIC son aquellas relacionadas con la obtención y producción de materiales directamente vinculados a las obligaciones diarias del aula como son: uso de la web para obtener recursos con el $75.76 \%$, elaboración de presentaciones en clase con el $69.70 \%$ y realización de trabajos asignados en clase también con el $69.70 \%$. Por otro lado, las actividades en que menos utilizan las TIC son: difusión de producciones personales con el $63.64 \%$, trabajos grupales fuera de clase con el $48.48 \%$ y la consulta de bibliotecas on-line para tareas también con el $48.48 \%$; que corresponden a tareas de mayor autonomía fuera del aula. Esto evidencia que no se ha logrado integrar las TIC de forma que se aproveche su potencial en el desarrollo de aprendizajes autónomos y de calidad, y que los estudiantes tengan seguridad de la calidad de sus trabajos como para publicarlos o compartirlos con los demás.

Figura 3. Frecuencia de uso de las TIC que hacen los estudiantes universitarios en diferentes actividades académicas.

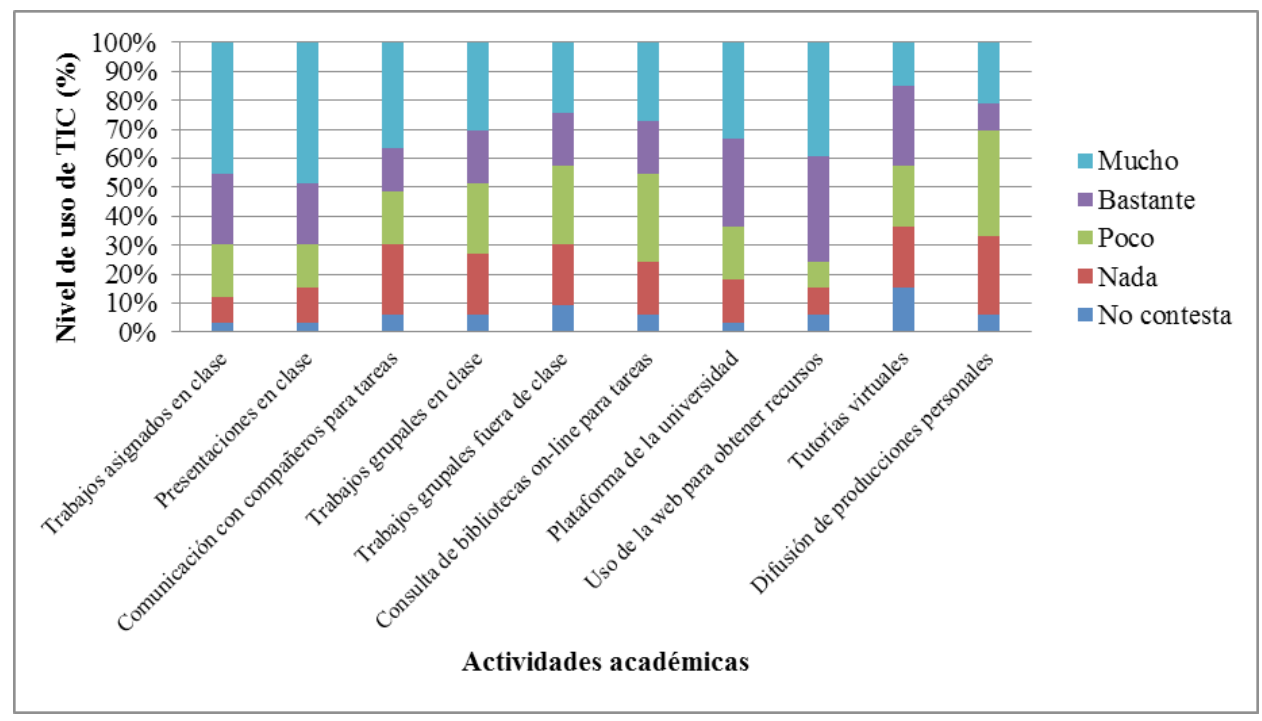

Fuente: elaboración propia.

Dentro de este apartado se pudo determinar que los estudiantes que utilizan más las TIC en sus diferentes actividades académicas son los comprendidos entre 29 a 35 años de edad, con un valor de 2.74. En cambio, el grupo que menos utilizan las TIC son los comprendidos entre 36 a 51 años, con un valor de 2.30. Aquí solamente se encontró una correlación negativa significativa indicando que conforme aumenta la edad de los estudiantes menos trabajan en equipo fuera de clase con el apoyo de las TIC. 
Tabla 5. Relaciones entre la edad y el tipo de uso que hacen de las TIC los estudiantes universitarios en sus estudios actuales

\begin{tabular}{|c|c|c|c|c|c|}
\hline Tipo de uso de las TIC & $\mathrm{N}$ & $\begin{array}{c}\text { Media } \\
\text { (nivel de uso) }\end{array}$ & $\begin{array}{c}\text { Desviación } \\
\text { estándar }\end{array}$ & $\begin{array}{l}\text { Coeficiente de correlación } \\
\text { (Rho de Spearman) }\end{array}$ & $\begin{array}{l}\text { Significatividad } \\
\text { (unilateral) }\end{array}$ \\
\hline $\begin{array}{l}\text { Trabajos asignados en } \\
\text { clase }\end{array}$ & 32 & 3.00 & 1.16 & 0.071 & 0.350 \\
\hline Presentaciones en clase & 32 & 3.03 & 1.20 & -0.153 & 0.201 \\
\hline $\begin{array}{l}\text { Comunicación con } \\
\text { compañeros para tareas }\end{array}$ & 32 & 2.53 & 1.39 & -0.108 & 0.278 \\
\hline $\begin{array}{l}\text { Trabajos grupales en } \\
\text { clase }\end{array}$ & 32 & 2.44 & 1.32 & -0.177 & 0.167 \\
\hline $\begin{array}{l}\text { Trabajos grupales } \\
\text { fuera de clase }\end{array}$ & 32 & 2.25 & 1.32 & -0.302 & 0.047 \\
\hline $\begin{array}{l}\text { Consulta de bibliotecas } \\
\text { online para tareas }\end{array}$ & 32 & 2.41 & 1.27 & -0.074 & 0.344 \\
\hline $\begin{array}{l}\text { Plataforma de la } \\
\text { universidad }\end{array}$ & 32 & 2.75 & 1.19 & 0.224 & 0.109 \\
\hline $\begin{array}{l}\text { Uso de la web para } \\
\text { obtener recursos }\end{array}$ & 32 & 2.94 & 1.22 & 0.037 & 0.421 \\
\hline Tutorías virtuales & 32 & 2.03 & 1.33 & -0.055 & 0.382 \\
\hline $\begin{array}{l}\text { Difusión de } \\
\text { producciones personales }\end{array}$ & 32 & 2.09 & 1.23 & -0.270 & 0.068 \\
\hline
\end{tabular}

Nota: Las correlaciones significativas al nivel de 0.05 (unilateral) están sombreadas.

Fuente: elaboración propia.

No se encontraron diferencias significativas en el tipo de uso de las TIC durante sus estudios actuales, según el sexo de los universitarios.

\section{DISCUSIÓN Y CONCLUSIONES}

Considerando que el objetivo establecido para este trabajo de investigación fue indagar la percepción respecto al uso de las TIC que tienen los estudiantes de primer ciclo en la carrera de Ciencias de la Educación de la Universidad Técnica Particular de Loja, los resultados fueron útiles para la determinación de fortalezas y potencialidades en sus conocimientos, usos y actitudes frente a estas tecnologías, así como de varios aspectos de mejora formativa que deberían ser tomados en cuenta por sus profesores y las autoridades universitarias responsables de garantizar su adecuada formación profesional. La concreción de estas acciones cobra importancia debido a que los estudiantes que inician una carrera en docencia y más aún en una modalidad abierta y a distancia requieren un buen manejo de las 
TIC, tanto como medio para el aprendizaje actual como para su futuro profesional. En este contexto, al igual que otras investigaciones sobre las percepciones de los estudiantes (Arain et al., 2019; Cabero-Almenara y Meza-Cano, 2019), el análisis de la información generada permite afirmar que los participantes en este estudio tienen una percepción muy positiva de los beneficios que proporciona el conocimiento y uso de las TIC, y dentro de ellas, particularmente las denominadas herramientas Web 2.0. Por ello, las vienen utilizando habitualmente en sus actividades académicas, aunque requieren mayor preparación en herramientas y aplicaciones específicas para el aprendizaje y el futuro profesional. A continuación, se detalla cómo los resultados sirvieron para responder las preguntas de investigación, comparándolos con hallazgos de investigaciones similares.

Respecto al nivel de conocimientos que poseen los estudiantes de la carrera de Ciencias de la Educación de la Universidad Técnica Particular de Loja, sobre las diferentes herramientas, recursos y aplicaciones tecnológicas, ellos consideran que tienen un mayor nivel de conocimiento de aquellas TIC que les permiten estar en contacto o interactuar con otras personas y conseguir información en general, como son los navegadores de internet, el correo electrónico y las redes sociales, pero lamentablemente tienen un conocimiento menor de aplicaciones tecnológicas más específicas para fines académicos como las bases de datos, las plataformas educativas, creación de mapas conceptuales, creación y publicación de contenidos digitales, etc. Esto evidencia que aún no se supera lo señalado por Esteve et al., (2014), quienes refieren varios estudios en donde se afirma que las nuevas generaciones se caracterizan por tener desarrolladas algunas habilidades TIC, pero que están relacionadas a actividades sociales y lúdicas, sin demostrar capacidad para transferirlas al aprendizaje ni a la construcción de conocimiento. Así mismo, en un trabajo más reciente con estudiantes de ingeniería de tres universidades colombianas y basándose en otras investigaciones, González y Gutiérrez (2017) indican que la generalización del conocimiento y uso de las TIC a nivel universitario aún sigue siendo superficial y tradicional. Siendo necesario que se realice un mayor número de estudios de las aplicaciones reales y no solo percepciones de las TIC, enfatizando las buenas prácticas docentes y estudiantiles, especialmente en el denominado aprendizaje móvil (Alonso-García et al., 2019; Romero-Rodríguez et al., 2020), lo que permitiría contar con mejores criterios y referencias del uso de las TIC en las universidades.

En el análisis adicional realizado con los mismos datos generados en el presente estudio, en donde se tomó en cuenta la edad y el sexo de los estudiantes, se pudo determinar que los más jóvenes y los varones tienen una mayor afinidad por algunas TIC. En el caso de los jóvenes, sus respuestas permitieron establecer una diferencia positiva significativa para el nivel de conocimientos en procesadores de texto, manejadores de presentaciones, navegadores de internet, redes sociales, repositorios digitales y publicaciones online de fotos. Y en trece de las veintidós TIC propuestas, los hombres indicaron tener un mayor conocimiento que las mujeres. Este tipo de diferencias también han sido encontradas en otros estudios como el de Torres-Coronas y Vidal-Blasco (2015), aunque dichas investigadoras insisten en la idea de que, dada la importancia actual de la competencia digital, no deberían existir brechas por sexo ni por grado universitario según el ámbito de estudio, opinión que según la evidencia estaría alejada de la realidad. Por otro lado, Cabero-Almenara y Meza-Cano (2019), no encontraron diferencias por sexo en cuanto a la percepción global que las herramientas Web 2.0 permiten obtener una enseñanza de calidad, pero una de las limitaciones de su estudio fue que no tomaron en cuenta usos específicos como: organizar, comunicar, crear, entre otros; lo cual podría marcar una diferencia, y sí fue abordado en el presente estudio. 
Al considerar la frecuencia de uso de las TIC que hacen en su aprendizaje, los estudiantes también señalaron que las herramientas, recursos y aplicaciones tecnológicas usadas con mayor frecuencia son las que les permiten interactuar con otras personas y conseguir información en general, especialmente los navegadores de internet, las redes sociales y el correo electrónico. En el caso específico del uso de las TIC para actividades académicas, consideran que las más utilizadas son las necesarias para cumplir con las obligaciones estudiantiles como obtener recursos de la web, elaborar presentaciones y realizar trabajos asignados en clase. Muy por debajo quedaron otros usos importantes en un contexto universitario como los relacionados con bases de datos generales y bibliográficas, bibliotecas online y trabajos grupales tanto dentro como fuera de la clase, ya que ellos les servirían para disponer de información científica actualizada y elaborar documentos académicos de calidad, entre otros beneficios. Esta ventaja de las TIC, es aprovechada de mejor forma en carreras universitarias propias de esta área, como lo demuestra el estudio de Humanante-Ramos, García-Peñalvo, Conde-González y Velasco-Silva (2015), en donde se encontró que los estudiantes de ingeniería en sistemas y computación a diferencia de otras carreras realizan un uso importante de Google Académico, bases de datos académicas y repositorios científicos, aunque su uso es combinado con buscadores genéricos, redes sociales y entretenimiento.

Esta falta de persistencia y pertinencia en el uso de las TIC para actividades académicas, también es consistente con estudios como el de Islas (2018), en donde aplicando el nuevo enfoque sistémico-conectivista se evidenció que el uso de las tecnologías aún no es completamente valorado como una fortaleza para el aprendizaje. Al respecto, Vinueza y Simbaña (2017) consideran que la escasa utilización que se hace del internet con fines de aprendizaje, se debe justamente a la falta de enseñanza de su uso adecuado como medio de aprendizaje. De forma, que es fundamental la determinación y difusión de las mejores prácticas de enseñanza utilizando TIC y la selección de los contenidos formativos, tanto como referencia para los procesos de capacitación de los demás profesores como para que los estudiantes universitarios aprendan a utilizarlas en sus carreras y en su futuro profesional (Cabero, 2014; Alonso-García, 2019; Romero-Rodríguez et al., 2020).

En cuanto a la relación entre el uso de las TIC y la edad de los estudiantes, se pudo determinar que conforme aumenta la edad, decae el uso del correo electrónico, los manejadores de presentaciones, navegadores de internet, sistemas de protección, redes sociales, repositorios digitales y publicaciones on-line de fotos. Así como también les agrada menos apoyarse en el uso de las TIC para realizar trabajos en equipo fuera de la clase, con una significatividad de 0,047. Estos resultados podrían deberse al tiempo que lleva cada estudiante interactuando con las TIC, y a las oportunidades de formación formal e informal que han tenido, en donde la ventaja es para los más jóvenes. En el análisis por sexo, se encontró que los hombres usan más los programas de chat y videoconferencias, y las publicaciones online de video para su aprendizaje. Lo cual, se corrobora parcialmente con estudios como el de Torres-Coronas y Vidal-Blasco (2015), quienes encontraron que los hombres se destacan en la comunicación en línea, pero en cambio las mujeres lo hacen en el manejo de redes sociales. Así mismo, Aranda et al. (2019) señala que, si bien no hay diferencias por sexo en los conocimientos básicos y medios de las TIC, sí se presentan diferencias cuando se necesitan conocimientos más complejos para su manejo. Estas diferencias podrían deberse al número y especialidad de los estudiantes encuestados, y al énfasis de cada instrumento de obtención de datos en unos u otros usos de las TIC. 


\section{REFERENCIAS BIBLIOGRÁFICAS}

Agencia de Regulación y Control de las Telecomunicaciones (2017). TIC y educación se analizaron en un Foro Internacional en Quito. Recuperado de http://bit.ly/2mfq7rQ

Alexander, B., Ashford-Rowe, K., Barajas-Murphy, N., Dobbin, G., Knott, J., McCormack, M. y Weber, N. (2019). EDUCAUSE Horizon Report: 2019 Higher Education edition. EDUCAUSE: Louisville.

Alonso-García, S., Aznar-Díaz, I., Cáceres-Reche, M. P., Trujillo-Torres, J. M. y RomeroRodríguez, J. M. (2019). Systematic review of good teaching practices with ICT in spanish higher education. Trends and challenges for sustainability. Sustainability, 11(24), 7150. doi: https://doi.org/10.3390/su11247150

Arain, A. A., Hussain, Z., Rizvi, W. H. y Saleem, M. (2019) Extending UTAUT2 toward acceptance of mobile learning in the context of higher education. Universal Access in the Information Society, (18), 659-673. doi: https://doi.org/10.1007/s10209-019-00685-8

Aranda, L., Rubio, L., Di Giusto, C. y Dumitrache, C. (2019). Evaluación del uso de las TIC en estudiantes de la Universidad de Málaga: diferencias de género. International Journal of Technology and Educational Innovation, 5(1), 63-71. doi:10.24310/innoeduca.2019.v5i1.5175

Brown, M., McCormack, M., Reeves, J., Brooks, D. C., Grajek, S., Alexander, B., Bali, M., Bulger, S., Dark, S., Engelbert, N., Gannon, K., Gauthier, A., Gibson, D., Gibson, R., Lundin, B., Veletsianos, G. y Weber, N. (2020). EDUCAUSE Horizon Report, Teaching and Learning Edition. EDUCAUSE: Louisville.

Cabero, J. (2010). Los retos de la integración de las TIC en los procesos educativos. Límites y posibilidades. Revista Perspectiva Educacional, 49(1), 32-61.

Cabero Almenara, J. (2014). Formación del profesorado universitario en TIC. Aplicación del método Delphi para la selección de los contenidos formativos. Educación XX1, 17(1), 111-132. doi: 10.5944/educxx1.17.1.10707.

Cabero-Almenara, J. y Martínez, A. (2019). Las Tecnologías de la Información y Comunicación y la formación inicial de los docentes. Modelos y competencias digitales. Profesorado. Revista de Currículum y Formación de Profesorado, 23(3), 247-268. doi:10.30827/profesorado. v23i3.9421

Cabero-Almenara, J. y Meza-Cano, J. (2019). Percepciones de estudiantes de licenciatura en línea sobre el impacto de la Web 2.0 en educación superior. Cultura y Educación. doi: $10.1080 / 11356405.2019 .1630953$

Cobo, C. y Moravec, J. (2011). Aprendizaje invisible. Hacia una nueva ecología de la educación. Barcelona: Laboratori de Mitjans Interactius/Publicacions i Edicions de la Universitat de Barcelona.

Cobo, C. (2016). La Innovación Pendiente. Reflexiones (y Provocaciones) sobre educación, tecnología y conocimiento. Colección Fundación Ceibal/Debate: Montevideo.

Durall, E., Gros, B., Maina, M., Johnson, L. y Adams, S. (2012). Perspectivas tecnológicas: educación superior en Iberoamérica 2012-2017. The New Media Consortium: Austin.

Esteve, F., Duch, J. y Gisbert, M. (2014). Los aprendices digitales en la literatura científica: diseño y aplicación de una revisión sistemática entre 2001 y 2010. Píxel-Bit. Revista de Medios y Educación, (45), 9-21. doi: http://dx.doi.org/10.12795/pixelbit.2014.i45.01

García, P. (2015). Desarrollo y evaluación de una experiencia piloto de mentoría con estudiantes de primer ciclo de educación superior a distancia, centro universitario asociado Quito. [Tesis de Licenciatura, Universidad Técnica Particular de Loja]. Repositorio Institucional - UTPL. Recuperado de http://bit.ly/2n9ZSUj

George, C. y Trujillo, L. (2018). Aplicación del Método Delphi Modificado para la Validación de un Cuestionario de Incorporación de las TIC en la Práctica Docente. Revista Iberoamericana de Evaluación Educativa, 11(1), 113-135. doi: https://doi.org/10.15366/riee2018.11.1.007 
González, R. y Gutiérrez, A. (2017). Competencias mediática y digital del profesorado e integración curricular de las tecnologías digitales. Revista Fuentes, 19(2), 57-67. doi: http://dx.doi. org/10.12795/revistafuentes.2016.19.2.04

Gros, B. y Contreras, D. (2006). La alfabetización digital y el desarrollo de competencias ciudadanas. Revista Iberoamericana de Educación, (42), 103-125.

Hamodi, C., López, V. M. y López, A. T. (2015). Medios, técnicas e instrumentos de evaluación formativa y compartida del aprendizaje en educación superior. Perfiles Educativos, 37(147), 146-161.

Herrera, M. (2019). Un modelo pedagógico para la educación superior virtual centrado en el aprendizaje colaborativo. En P. Vélez y Y. Yaguana (Eds.), Nuevas tecnologías en el proceso de enseñanza-aprendizaje. Universidad Técnica Particular de Loja: Loja.

Humanante-Ramos, P., García-Peñalvo, F., Conde-González, M. y Velasco-Silva, D. (2015). Diagnóstico del uso de los dispositivos electrónicos y de las herramientas web 2.0 desde un enfoque PLE, en un grupo de estudiantes de ingeniería. En III Congreso Internacional sobre Aprendizaje, Innovación y Competitividad (pp. 284-289). Recuperado de http://bit.ly/2mnWiWi

Instituto Nacional de Estadística y Censos (2016). Tecnologías de la Información y Comunicaciones. Quito. Recuperado de http://bit.ly/2n8fVln

Islas, C. (2018). Implicación de las TIC en el aprendizaje de los universitarios: una explicación sistémico conectivista. Píxel-Bit. Revista de Medios y Educación, (52), 199-215. doi: http:// dx.doi.org/10.12795/pixelbit.2018.i52.14

Johnson, L., Adams, S., Gago, D., García, E. y Martín, S. (2013). Perspectivas Tecnológicas: Educación Superior en América Latina 2013-2018. Un Análisis Regional del Informe Horizon NMC. The New Media Consortium: Austin.

López-Gómez, E. (2018). El método Delphi en la investigación actual en educación: una revisión teórica y metodológica. Educación XX1, 21(1), 17-40. doi: 10.5944/educXX1.20169

Martínez, M. E. y Raposo, M. (2006). Las TIC en manos de los estudiantes universitarios. Revista Latinoamericana de Tecnología Educativa, 5(2), 165-176.

Organización de las Naciones Unidas para la Educación, la Ciencia y la Cultura. (2011). UNESCO ICT Competency Framework for Teachers. París. Recuperado de http://bit.ly/2oyW0fL

Ortiz, A. M., Peñaherrera, M. y Ortega, J. M. (2012). Percepciones de profesores y estudiantes sobre las TIC. Un estudio de caso. Edutec. Revista Electrónica de Tecnología Educativa, (41), 1-15. doi: https://doi.org/10.21556/edutec.2012.41.352

Palacios, A. y López-Pastor, V. (2013). Haz lo que yo digo pero no lo que yo hago: sistemas de evaluación del alumnado en la formación inicial del profesorado. Revista de Educación, 361, 279-305. doi: 10.4438/1988-592X-RE-2011-361-143.

Perazzo, M. (2008). La ruta de la alfabetización digital en la educación superior: una trama de subjetividades y prácticas. Revista de Universidad y Sociedad del Conocimiento, 5(1), 1-10.

Prendes, M. P. (Dir.). (2010). Competencias TIC para la docencia en la Universidad Pública Española: Indicadores y propuestas para la definición de buenas prácticas: Programa de Estudio y Análisis. Informe del Proyecto EA2009-0133 de la Secretaría de Estado de Universidades e Investigación. Universidad de Murcia y Ministerio de Educación: Murcia.

Raposo, M., Martínez, M. E., Añel, M. E., Doval, M. I., Rodríguez, J., Pereira, M. C. y González, F. (2012). A presenza e emprego das TIC no ensino obrigatorio: o caso da provincia de Ourense. En Raposo, M. y Martínez M. E. (Coord.), As TIC e as súas aplicacións na aula: misión posible (pp. 295-314). Noia: Toxosoutos.

Rodríguez, R. (2010). El impacto de las TIC en la transformación de la enseñanza universitaria: Repensar los modelos de enseñanza y aprendizaje. Teoría de la Educación: Educación y Cultura en la Sociedad de la Información, 11(3), 32-68.

Romero-Rodríguez, J. M., Aznar-Díaz, I., Hinojo-Lucena, F. y Cáceres-Reche, M. (2020). Models of good teaching practices for mobile learning in higher education. Palgrave Communications, 80(6). doi: https://doi.org/10.1057/s41599-020-0468-6 
Torres-Coronas, T. y Vidal-Blasco, M. (2015). Percepción de estudiantes y empleadores sobre el desarrollo de competencias digitales en la Educación Superior. Revista de Educación, (367), 63-90. doi: 10.4438/1988-592X-RE-2015-367-283

Vinueza, S. y Simbaña, V. (2017). Impacto de las TIC en la Educación Superior en el Ecuador. Revista Publicando, 4(11), 355-368. 